\title{
DEPENDÊNCIA QUÍMICA E O CENÁRIO DOS USUÁRIOS DE DROGAS ATENDIDOS NOS CENTROS DE ATENÇÃO PSICOSSOCIAL ÁLCOOL E DROGAS: A POLÍTICA DE SAÚDE NA ATENÇÃO INTEGRAL AOS IDOSOS
}

\author{
CHEMICAL DEPENDENCY AND THE SCENARIO OF DRUG USERS TREATED AT THE \\ PSYCHOSOCIAL CARE CENTERS FOR ALCOHOL AND DRUGS: HEALTH POLICY IN \\ COMPREHENSIVE CARE FOR THE ELDERLY
}

\author{
Aldo Pacheco Ferreira \\ Escola Nacional de Saúde Pública Sergio Arouca (ENSP/Fiocruz) \\ E-mail: aldopachecoferreira@gmail.com
}

\section{RESUMO}

O uso de drogas lícitas e ilícitas, se constitui em enorme desafio para a saúde pública, no entanto, com o aumento da expectativa de vida da população brasileira, essa realidade também se faz presente em idosos. Investigou-se o uso de drogas lícitas e ilícitas, o tempo de uso, as associações destas drogas com transtornos mentais, e o perfil sociodemográfico dos idosos frequentadores dos Caps-AD, Município do Rio de Janeiro, que nasceram até 31 de dezembro de 1958 e que procuraram atendimento no período de 2001 a dezembro de 2019. Trata-se de um estudo com delineamento descritivo, analítico observacional de temporalidade retrospectiva, baseado em dados secundários. Foi feita inicialmente uma análise descritiva da população estudada a partir do cálculo de medidas de tendência central e dispersão para variáveis contínuas e de frequência para variáveis categóricas. A associação das variáveis dependentes com as variáveis explicativas foi investigada através da estimativa das odds ratio (OR) simples e ajustadas. A significância estatística foi avaliada através do teste de Tukey (ANOVA). Verificaram-se maiores percentuais de homens (76,62\%), com idade entre 60 e 73 anos (87,4\%), sem companheiro(a) (64,35\%), com ensino fundamental (67,59\%), desempregados $(32,87 \%)$ e em tratamento entre 1 e 5 anos $(69,21 \%)$. As drogas de maior uso foram: o álcool, a maconha, o crack e a cocaína. Evidencia-se necessidade de maior conhecimento sobre dependência química na terceira idade e uso de estratégias e aplicação de instrumentos que facilitem a identificação precoce desses usuários.

Palavras-chave: Idosos; Transtornos relacionados ao uso de Substâncias; Saúde mental; Drogas; Direitos humanos.

\section{ABSTRACT}

The use of legal and illegal drugs is a massive challenge for public health; however, with the increase in life expectancy for the Brazilian population, this reality is also present in the elderly. The use of licit and illicit drugs was investigated, the time of use, the associations of these drugs with mental disorders, and the sociodemographic profile of the elderly who attended at Caps-AD, Municipality of Rio de Janeiro, who were born until December 31 from 1958 and who sought care in the period from 2001 to December 2019. This is a study with a descriptive, analytical observational design of retrospective temporality, based on secondary data. Initially, a descriptive analysis of the studied population was made based on the calculation of measures of central tendency and dispersion for continuous variables and frequency for categorical variables. The association of dependent variables with explanatory variables was investigated by estimating simple and adjusted odds ratios (OR). Statistical significance was assessed using the Tukey test (ANOVA). There were higher percentages of men (76.62\%), aged between 60 and 73 years (87.4\%), without a partner $(64.35 \%)$, with elementary education $(67.59 \%)$, unemployed $(32.87 \%)$, and undergoing treatment between 1 and 5 years $(69.21 \%)$. The most used drugs were: alcohol, marijuana, crack, and cocaine. There is a need for greater knowledge about chemical dependency in the elderly and the use of strategies and the application of instruments that facilitate the early identification of these users.

Keyword: Elderly; Disorders related to Substance use; Mental health; Drugs; Human rights. 


\section{INTRODUÇÃO}

O idoso, na atualidade, com suas adequações e integração social tem exigido atenção especial, considerando que o processo de envelhecimento saudável envolve desafios, reflexões e suporte às demandas que o processo em si requer. $\mathrm{O}$ aumento da longevidade e das condições de saúde tem contribuído diretamente para o aumento constante da população idosa (CRUZ et al., 2016). Globalmente, adultos com 60 anos ou mais passarão de 516 milhões em 2009, para um total de aproximadamente 1,43 bilhões em 2050 (DESTRO, 2018). Segundo Miranda et al. (2016), tal transformação no perfil demográfico ocasiona novos desafios para a saúde pública, não somente pelas demandas crescentes, mas ainda pela forma como os padrões de comportamento das gerações se têm alterado.

As políticas de atenção ao usuário de álcool e outras drogas são fundamentadas na reforma da assistência psiquiátrica no Brasil, que é baseada na lei 10.216 (ALMEIDA, 2019). Essas políticas são baseadas na garantia dos direitos dos usuários do Sistema Único de Saúde (SUS) e estabelece como diretrizes, a universalidade do acesso, o direito à assistência, integralidade, descentralização e territorialização, que objetivam ações no âmbito psicossocial, favorecendo a autonomia do sujeito e a vida social (FERREIRA et al., 2015).

O uso de drogas está propriamente conexo à numerosos prejuízos de ordem física, mental, ocupacional e social, e de tal forma traduz-se tanto para o usuário, quanto para sua família e sociedade, que repercutem em grave problema de saúde pública (KUERBIS et al., 2014). Historicamente, houve pouca discussão relacionada à temática sobre idosos que fazem uso abusivo de álcool e drogas ilícitas, provocando, por conseguinte, o desenvolvimento de ações que possam contribuir à prevenção e à promoção de saúde, com as políticas públicas que urgem ao fato (FEITOSA et al., 2016). Ademais, os idosos são mais vulneráveis aos efeitos iatrogênicos do consumo de drogas e a dependência devido as modificações na composição corporal, aumento da morbidade e alta utilização de medicamentos psicoativos. Por conseguinte, a dependência é, muitas vezes, confundida com depressão ou demência (CRUZ et al., 2016).

As diversas maneiras de compreender o sofrimento mental assumiram em cada período histórico uma significação específica relacionada à ordem social vigorante. Discursos foram propagados, tratamentos e espaços sociais foram impostos aos ditos "loucos", "os anormais", "os alienados". O tipo de assistência destinada a estes sujeitos baseou-se por um longo período numa lógica segregacionista pautada na perspectiva asilar (DEL'OLMO; CERVI, 2017). Na década de 1980, o movimento da Reforma Psiquiátrica no Brasil fomentou a proposta de consolidar uma rede de cuidados em saúde mental que não se paute na centralidade do hospital psiquiátrico. O padrão de tratamento em saúde mental em vigência era basicamente hospitalocêntrico e admitia pouco a dimensão da experiência no seguimento do indivíduo. A internação não se alinhava com uma demanda clínica da pessoa, mas sim, com uma demanda da sociedade, institucionalizando o sujeito dentro de uma política que se mostrou segregacionista e punitiva. Esse modelo manicomial gerava diferentes efeitos nocivos às pessoas, tais como: progressiva desconexão com seus laços sociofamiliares e perda das habilidades para atividades da vida diária (SANTOS et al., 2020).

A Lei Federal $n^{\circ}$ 10.216/2001 instituiu a Reforma Psiquiátrica Brasileira, de forma a redirecionar a assistência em saúde mental, proporcionando atendimentos nas bases comunitárias do usuário desse serviço (BRASIL, 2001). Desde então, uma nova política de saúde mental foi instituída no país e teve como um dos seus principais recursos os Centros de Atenção Psicossocial (Caps), dentre esses o Centro de Atenção Psicossocial Álcool e Drogas (Caps-AD), local onde o tratamento se desenvolve de forma comunitária e compartilhada, reorientando o cuidado dos usuários problemáticos de álcool e outras drogas. Com efeito, possibilitou objetivar o modelo de atenção à pessoa com transtorno mental, implementando nos serviços de saúde mental um acesso livre, contrário as longas internações perpetradas por vários anos nos antigos hospitais psiquiátricos e manicômio (CALDAS, 2019).

Destaca a Organização Mundial de Saúde (OMS) está o consumo de álcool entre os principais fatores de risco para o desenvolvimento de Doenças Crônicas Não Transmissíveis (DCNT) (doenças cerebrovasculares e cardiovasculares, neoplasias, obesidade, diabetes, hipertensão, entre outras). Ademais, está associado ao risco de desenvolvimento de distúrbios mentais e comportamentais, bem como lesões resultantes de violência e acidentes de trânsito (NORONHA et al., 2019).

O consumo imoderado de bebidas alcóolicas é considerado como uma epidemia, sendo a terceira causa de mortes no mundo, atrás somente das doenças cardiovasculares e do câncer (WHO, 2013). Além disso, a ingestão excessiva de álcool é caracterizada pela 
progressão de doenças agudas e por afetar negativamente as relações pessoais, sociais e a qualidade de vida (KUERBIS et al., 2014, ZHANG et al., 2020). Os resultados obtidos do primeiro levantamento sobre os padrões de consumo de álcool em idosos no Brasil, evidenciaram que $12 \%$ dos idosos bebiam pesado (mais de 7 doses por semana), 10,4\% bebiam em excesso (mais de 3 doses em uma ocasião) e 2,9\% dependiam do álcool (CASTRO-COSTA et al., 2008). Posteriores estudos registraram uma variação entre $1,1 \%$ e $12,4 \%$, levando-se em conta outros padrões de consumo (moderado, excessivo, pesado, binge, abusivo e dependência) (NORONHA et al., 2019).

Cabe destacar, que o uso de substância psicoativa em idosos ainda é pouco explorado. $\mathrm{O}$ número de idosos atendidos em serviços especializados para o tratamento do uso de substâncias psicoativas é baixo, o que leva a repensar a prática assistencial que tem sido oferecida pelos profissionais de saúde (PILLON et al., 2010). Além disso, muitas prescrições medicamentosas utilizadas por essa população interagem de modo adverso com o consumo de substâncias psicoativas, seja anulando, seja potencializando seus efeitos (SILVA; HERZOG, 2015). Os utilizadores de drogas continuam a morrer mais cedo comparativamente à população geral, e os tipos de mortes não relacionadas ao uso de substâncias mais comuns são doenças hepáticas, neoplasias, infeções crônicas respiratórias inferiores e hepatites. Essas condições mórbidas, apesar de não estarem classificadas como tal, podem estar associadas ao uso nocivo de substâncias (DESTRO, 2018).

As políticas de atenção ao idoso usuário de álcool e outras drogas, conquanto seja demonstrado um índice baixo de idosos que usam substâncias psicoativas, apontam Noronha et al. (2019) que as pesquisas podem estar limitadas por falta de informações mais específicas nessa população. Destro (2018) ressalta, ainda, a necessidade de mais estudos com vistas a elucidar o perfil desta clientela e o progresso da exposição ao uso problemático, bem como documentar fatores de risco e proteção associados ao uso, abuso e dependência das substâncias lícitas e ilícitas, de forma que possam trazer entendimentos necessários para o desenvolvimento de trabalhos assistenciais, educacionais e preventivos para esses clientes, bem como o desenvolvimento de instrumentos que ajudem os profissionais de saúde a identificar e intervir precocemente em tais riscos.

Assim sendo, este estudo tem como objetivo investigar o uso de drogas lícitas e ilícitas, o tempo de uso, as associações destas drogas com transtornos mentais, e o perfil sociodemográfico dos clientes idosos atendidos nos
Caps-AD, instalados no Município do Rio de Janeiro, no período de 2001 a 2019.

\section{METODOLOGIA}

Trata-se de um estudo com delineamento descritivo, analítico observacional de temporalidade retrospectiva, baseado em dados secundários, com abordagem quantitativa do tipo transversal, que teve como público-alvo os usuários de álcool e/ou outras drogas que nasceram até 31 de dezembro de 1958 e que procuraram atendimento no período de 2001 a dezembro de 2019.

Segundo Baptista; Campos (2016), o pesquisador que conduz investigação descritiva observa, conta, descreve e classifica e quando a descrição é quantitativa envolve a predominância, incidência, o tamanho e os atributos mensuráveis de um fenômeno. Já o estudo exploratório investiga a natureza do fenômeno e outros fatores com os quais está relacionado.

Quando se trata de estudo observacional com delineamento transversal analisa-se indivíduos em um mesmo momento histórico, bem como o fator e o efeito da exposição, identificando a existência de associações entre exposição e doença (GIL, 2008). Há também a inferência que é o conjunto de técnicas, assentes na teoria das probabilidades, que permitem construir proposições de caráter probabilístico acerca da população, partindo da observação de alguns dos seus elementos-amostra (MOORE, 2000).

Os sujeitos desta pesquisa foram os usuários do Caps-AD. Os dados para análise do perfil clínico e sociodemográfico foram obtidos nos prontuários médicos arquivados nos Caps-AD foram estabelecidos os seguintes critérios de inclusão: a) pacientes com idade igual ou superior a 60 anos; b) que tiveram no mínimo um atendimento por uso abusivo de álcool e/ou outras drogas; c) com os dados registrados nos prontuários completos e legíveis.

No período da coleta dos dados, para a realização do cálculo e do sorteio da amostra, foi efetivado uma verificação dos clientes idosos que estavam em atendimento nos Caps-AD. Para a realização do sorteio amostral foi utilizado os recursos do Programa SPSS Statistics 24. Para cálculo do tamanho amostral foi considerada uma prevalência de uso de múltiplas substâncias psicoativas de 39,0\%, uma precisão de $4 \%$ e um intervalo de confiança de 95\% (TREVISAN; CASTRO, 2019). Para uma população finita de 1107 clientes dos serviços, obteve-se o tamanho amostral de 432 pacientes que procuram o serviço de reabilitação 
psicossocial com alguma queixa decorrente do uso abusivo de substâncias lícitas ou ilícitas. A coleta dos dados foi realizada no período compreendido entre novembro/2020 e janeiro de 2021.

As variáveis investigadas nos prontuários foram: sexo, idade, estado civil, escolaridade, situação de trabalho, filhos, suporte familiar, tipo de demanda ao serviço de reabilitação, tipos de substâncias consumidas e adesão ao tratamento, este por sua vez segue os critérios temporais dos Caps-AD (PELISOLI; MOREIRA, 2007), onde se caracteriza como abandono ao tratamento aquele indivíduo que se afasta por seis meses ou mais de forma ininterrupta do serviço de atenção de reabilitação psicossocial.

Foi feita inicialmente uma análise descritiva da população estudada a partir do cálculo de medidas de tendência central e dispersão para variáveis contínuas e de frequência para variáveis categóricas. As estimativas de prevalência e respectivos intervalos de $95 \%$ de confiança foram obtidos aplicando-se pesos que corrigiram para o efeito de amostragem complexa.

A associação das variáveis dependentes com as variáveis explicativas foi investigada através da estimativa das odds ratio (OR) simples e ajustadas. A significância estatística foi avaliada através do teste de Tukey (ANOVA). A análise da significância estatística é considerada um procedimento para verificar a discrepância de uma hipótese estatística em relação aos dados observados, utilizando uma medida de evidência (p-valor). A análise de variância de um fator, ANOVA, é uma extensão do teste $\mathrm{t}$; e, dessa forma, permite verificar o efeito de uma variável independente qualitativa em uma variável dependente quantitativa. De acordo com Fávero et al. (2009), a hipótese consiste em testar se as médias das populações são iguais. Assim, cada grupo inclui as observações da variável dependente em uma categoria do fator. O nível de significância para todos testes foi de 5\%.

Quanto aos aspectos éticos da pesquisa, foram observados os disciplinados pela Resolução 466/2012 do Conselho Nacional de Saúde Ministério da Saúde (BRASIL, 2012). Seguiu de modo criterioso e ético as necessidades específicas para a realização de uma pesquisa com seres humanos, sobretudo quanto a questões de voluntariedade e confidencialidade. A pesquisa foi aprovada pelo Comitê de Ética em Pesquisa por meio da Plataforma Brasil, com número do parecer 3.461 .055 e CAAE 16828919.3.0000.5240.

\section{RESULTADOS}

Dentre as informações sociodemográficas, a média de idade foi de 66,2 anos $(\mathrm{DP} \pm 3,16)$, com variação de 60 a 76 anos. Conforme tabela 1, observou-se na população estudada um maior percentual de representação masculina $(77,2 \%)$, sem companheiro(a) (64,3\%), com ensino fundamental incompleto $(44,6 \%)$ e desempregados $(32,97 \%)$. Cabe destacar que a associação entre os pesquisados apresentou valor significativo para as variáveis sexo (p-valor 0,002 ) e idade (p-valor 0,001).

Tabela 1. Perfil sociodemográfico dos clientes idosos atendidos nos Caps-AD, Município do Rio de Janeiro, Brasil, 2021

\begin{tabular}{lccc}
\hline \multirow{2}{*}{ Variáveis/Categorias } & \multicolumn{2}{c}{ Distribuição } & p-valor \\
\cline { 2 - 4 } & $\mathbf{n = 4 3 2}$ & $\mathbf{\%}$ & 0,002 \\
\hline Sexo & & & \\
\hline Masculino & 331 & 76,62 & 0,001 \\
Feminino & 101 & 23,38 & \\
\hline Idade (anos) & 356 & & 0,513 \\
\hline De 60 a 70 & 76 & 17,4 & \\
De 71 a 80 & & & \\
\hline Estado civil & 65 & 15,05 & \\
\hline Solteiro & 98 & 22,69 & \\
Casado & 56 & 12,96 & 0,412 \\
União estável & 165 & 38,19 & \\
Viúvo & 48 & 11,11 & \\
Divorciado & & & \\
\hline Escolaridade & 13 & 3,0 & \\
\hline Sem instrução & 292 & 67,6
\end{tabular}


Ensino médio

Ensino superior incompleto

Ensino superior completo

\section{Ocupação trabalhista}

Carteira assinada

Autônomo

Desempregado

Aposentado

Em relação ao consumo de drogas, o maior quantitativo de uso foi de sustâncias lícitas (72\%) comparadas a ilícitas (28\%) (p-valor 0,222). Não houve diferenças significativas quanto a maiores chances de consumo de drogas lícitas e/ou ilícitas para homens $(\mathrm{OR}=$ 0,93; IC96) e mulheres ( $O R=1,21)$ (p-valor 0,000). Não foi constatada diferença significativa entres o sexo masculino $(\mathrm{OR}=1,13)$ e feminino $(\mathrm{OR}=0,77)$ quanto ao

\begin{tabular}{ccc}
115 & 26,62 & \\
9 & 2,08 & \\
3 & 0,7 & 0,525 \\
& & \\
68 & 15,74 & \\
15 & 3,47 & \\
142 & 32,87 & \\
207 & 47,9 & \\
\hline
\end{tabular}

uso de múltiplas drogas. Na população masculina $(\mathrm{n}=331)$, o álcool foi à droga mais utilizada seguida do tabaco, crack, maconha, êxtase, cocaína, anfetamina, benzodiazepínicos e demais drogas (ex: inalantes, merla, solventes). Na população feminina $(n=101)$, o tabaco foi à droga mais utilizada seguida do álcool, êxtase, anfetamina, crack, benzodiazepínicos, maconha, cocaína e demais drogas, conforme tabela 2 e tabela 3.

Tabela 2. Drogas utilizadas, segundo sexo, por clientes idosos atendidos nos Caps-AD, Município do Rio de Janeiro, Brasil, 2021

\begin{tabular}{lcccc}
\hline \multirow{2}{*}{ Drogas } & \multicolumn{3}{c}{$\mathbf{n = 4 3 2}$} & \multicolumn{2}{c}{ Mulheres $(\mathbf{n = 1 0 1} \mathbf{- 2 3 , 3 8 \% )}$} \\
\cline { 2 - 5 } & \multicolumn{2}{c}{ Homens $(\mathbf{n = 3 3 1} \mathbf{- 7 6 , 6 2 \% )}$} & $\mathbf{n}$ & $\mathbf{\%}$ \\
\cline { 2 - 5 } & $\mathbf{n}$ & $\mathbf{\%}$ & 73 & 72,28 \\
Tabaco & 154 & 77,04 & 58 & 57,42 \\
Álcool & 255 & 25,98 & 17 & 16,83 \\
Maconha & 86 & 16,01 & 12 & 11,88 \\
Cocaína & 53 & 27,49 & 33 & 32,67 \\
Crack & 91 & 14,8 & 43 & 42,57 \\
Anfetamina & 49 & 9,67 & 21 & 20,79 \\
Benzodiazepínico & 32 & 17,82 & 44 & 43,56 \\
Êxtase (MMDA) & 59 & 6,95 & 11 & 10,89 \\
Outras (ex: inalantes, & 23 & & & \\
merla, solventes) & & & & \\
\hline
\end{tabular}

Tabela 3. Distribuição de frequência e porcentagem em relação ao tipo de drogas utilizadas $(n=342)$ dos clientes idosos do Caps-AD, Município do Rio de Janeiro, Brasil, 2021

\begin{tabular}{lcc}
\hline \multicolumn{1}{c}{ Drogas } & n=432 & Percentual (\%) \\
\hline Tabaco & 65 & 15,05 \\
Álcool & 165 & 38,19 \\
Maconha & 9 & 2,08 \\
Cocaína & 3 & 0,7 \\
Crack & 48 & 11,11 \\
Anfetamina & 3 & 0,7 \\
Benzodiazepínico & 13 & 3,0 \\
Êxtase (MMDA) & 9 & 2,08 \\
Álcool, cocaína ou crack & 18 & 4,16 \\
Álcool, cocaína, crack e maconha & 16 & 3,7 \\
Álcool, crack, maconha e solventes & 7 & 1,63
\end{tabular}


Álcool, tabaco e maconha

66

15,28

Álcool, tabaco e êxtase

Álcool, tabaco, maconha e anfetamina

6

1,39

4

0,93

Os resultados expressam o maior percentual de idosos em tratamento no período entre 1 e 5 anos $(69,21 \%)$. Já $74,3 \%$ dos idosos foram encaminhados até os Caps-AD, com procura espontânea ao atendimento $(19,91 \%)$, encaminhamento por hospital psiquiátrico $(33,56 \%)$, seguidos pelas Unidades de Pronto Atendimento (UPAS) (30,79\%). Quanto ao quantitativo de clientes idosos dos Caps-AD que já haviam sido internados por uso de substâncias psicoativas, obteve-se o percentual de $51,16 \%$. E quanto aos que indicaram fazer uso de medicações relacionadas aos problemas decorrentes do uso das substâncias o indicativo foi de $33,1 \%$. Por fim, no tocante a modalidade de tratamento mais utilizada nos Caps-AD, obteve-se o percentual de $80,79 \%$, consonante a modalidade semi-intensiva (tabela $4)$.

Tabela 4. Dados de tempo de tratamento, acesso ao atendimento, tipologia e modalidade de encaminhamento recebido e usos de psicoativos por clientes idosos atendidos nos Caps-AD, Município do Rio de Janeiro, Brasil, 2021

\begin{tabular}{lcc}
\hline \multirow{2}{*}{ Variáveis/Categorias } & \multicolumn{2}{c}{ Distribuição } \\
\cline { 2 - 3 } Tempo de tratamento nos Caps (anos) & $\mathbf{n}$ & $\mathbf{4 3 2}$ \\
\hline Entre 1 e 5 & 299 & 69,21 \\
Entre 6 e 10 & 133 & 30,79 \\
Acima de 11 & - & \\
\hline Acesso ao atendimento & 86 & 19,91 \\
\hline Procura espontânea & 321 & 74,3 \\
Encaminhamento & 16 & 3,71 \\
Trabalho & 9 & 2,08 \\
Ordem judicial & & 30,79 \\
Tipologia de encaminhamento & 133 \\
\hline Unidades de Pronto Atendimento (UPAS) & 23 & 5,32 \\
Programa de Saúde da Família (PSF) & 39 & 9,03 \\
Unidades de emergência & 32 & 7,41 \\
Centros de Referência de Assistência Social (CRAS) & 49 & 11,34 \\
Grupos de apoio & 145 & 33,56 \\
Hospital psiquiátrico & 11 & 2,55 \\
Ordem judicial & & \\
\hline Modalidade de atendimento nos Caps-AD & 64 \\
\hline Intensivo & 349 & 14,81 \\
Semi-intensivo & 19 & 80,79 \\
Não intensivo & & 4,4 \\
\hline Faz uso de medicação devido ao uso de substância psicoativa & 143 \\
\hline Sim & 289 & 33,1 \\
Não & & 66,9 \\
\hline Internação por uso de substância psicoativa & 221 \\
\hline Sim & 211 & \\
\hline Não & & 51,16 \\
\hline
\end{tabular}

Dos diagnósticos clínicos, consolidados nas categorias da Classificação Internacional de Doenças (CID-10), os mais prevalentes foram do grupo da esquizofrenia, transtornos esquizotípicos e delirantes, com 176 clientes idosos (40,74\%), seguidos do grupo de retardo mental, presentes em 95 clientes idosos $(21,99 \%)$, e do grupo dos transtornos do humor [afetivos], com 65 clientes idosos $(15,04 \%)$ (tabela 5). 
Tabela 5. Distribuição segundo o diagnóstico clínico dos clientes idosos atendidos nos Caps-AD, Município do Rio de Janeiro, Brasil, 2021

\begin{tabular}{|c|c|c|}
\hline Código Internacional de Doenças 10 ${ }^{\text {a }}$ edição (CID-10) & $\mathbf{N}$ & $\%$ \\
\hline F00-F09: Transtornos mentais orgânicos, inclusive os sintomáticos & 30 & 6,94 \\
\hline $\begin{array}{l}\text { F10 a F19: Transtornos mentais e comportamentais devido ao uso de } \\
\text { substância psicoativa }\end{array}$ & 15 & 3,48 \\
\hline F20 a F29: Esquizofrenia, transtornos esquizotípicos e transtornos delirantes & 176 & 40,74 \\
\hline F30 a F39: Transtornos do humor [afetivos] & 65 & 15,04 \\
\hline $\begin{array}{l}\text { F40 a F48: Transtornos neuróticos relacionados com o estresse e } \\
\text { somatoformes }\end{array}$ & 36 & 8,33 \\
\hline F70-F79: Retardo mental & 95 & 21,99 \\
\hline Não definido & 15 & 3,48 \\
\hline Total & 432 & 100 \\
\hline
\end{tabular}

\section{DISCUSSÃO}

Este estudo examinou o uso de drogas lícitas e ilícitas, o tempo de uso, as associações destas drogas com transtornos mentais, e o perfil sociodemográfico dos clientes idosos atendidos nos Caps-AD do Município do Rio de Janeiro.

Dos clientes atendidos no serviço especializado em tratamento aos clientes idosos usuários de substâncias psicoativas, apresentam-se com alta prevalência de pacientes do sexo masculino se assemelhando com outros estudos nacionais (GONÇALVES; NUNES, 2014; SANTANA et al., 2020). Em considerando o diagnóstico principal do paciente no acolhimento, a maioria possuía esquizofrenia, transtornos esquizotípicos e transtornos delirantes (CID-10, F20 a F29), seguido de retardo mental (CID-10, F70-F79).

Dentre as informações sociodemográficas, a média de idade foi de 66,2 anos, com variação de 60 a 76 anos, com 64,3\% sem companheiro(a), 44,6\% com ensino fundamental incompleto e $32,97 \%$ desempregados. De acordo com Pope et al. (2010), o status socioeconômico influencia a experiência subjetiva do envelhecimento. Quanto mais baixo mais rápido são os eventos de vida que levam ao uso de drogas. Isso, combinado com fatores como violência e discriminação racial. Ademais, problemas financeiros devido à perda de emprego ou aposentadoria, perda do cônjuge e amigos, e isolamento social são causas conhecidas de depressão, que por sua vez podem desencadear o uso de substâncias lícitas e/ou ilícitas em idosos (PELISOLI; MOREIRA, 2007).

Historicamente, o uso de substâncias psicoativas está estritamente relacionado aos indivíduos mais jovens e tende a diminuir com o passar dos anos, porém estudos apontam que a idade nem sempre é um fator protetor contra o uso abusivo de drogas, além disso, frequentemente casos de idosos dependentes químico não são relatados e são subnotificados. Esses fatos ajudaram a perpetuar um equívoco de que idosos não fazem uso abusivo de drogas.22,34,35 Assim, uma das grandes dificuldades a serem enfrentada pelo Brasil é justamente os parâmetros sociais que serão levados em considerações para o processo de identificação, justamente por falta de um maior aprofundamento nos estudos voltados à saúde da população idosa.

O mito de que os idosos não usam drogas, quer lícitas ou ilícitas, e/ou não as usam de forma problemática foi dissipado. Assim, embora o uso de drogas ilícitas entre idosos seja considerado raro, estima-se que os números aumentem no futuro (WANG; ANDRADE, 2013). As razões para essa prevalência decrescente podem estar relacionadas à falta de autorrelato devido ao declínio cognitivo, problemas em distinguir problemas relacionados a substâncias de problemas de saúde e, finalmente, relutância em admitir o problema. Da mesma forma, o aumento da mortalidade entre os indivíduos com história uso de drogas ao longo da vida também pode contribuir para o declínio constante. Os números são baixos, em parte também porque não existem intervenções específicas que visem encorajar os idosos a participarem do tratamento (BEYNON, McVEIGH, ROE, 2007).

Cabe destacar que o uso de drogas tem sido associado ao início precoce do diabetes e ao agravamento de outras doenças relacionadas à idade, como doenças neurológicas, respiratórias e câncer. O efeito alucinante ou desorientador dos medicamentos aumentou o risco de queda e lesão acidental, que é uma das principais razões para incapacidade e mortalidade em idosos (ROE et al., 2010). Foi encontrada uma relação significativa entre quedas e uso de drogas psicoativas entre idosos. Lesões relacionadas a quedas são uma causa significativa de 
hospitalização, colocação em lares de idosos ou diminuição da mobilidade entre adultos mais velhos, respondendo por $40 \%$ das lesões acidentais nesta população (BEYNON, McVEIGH, ROE, 2007).

Os idosos usuários de substâncias podem não apresentar os mesmos sintomas que os mais jovens e, portanto, podem ser mais difíceis de identificar. As opções de tratamento permanecem geralmente limitadas, uma vez que poucos programas ou estabelecimentos de saúde oferecem intervenções personalizadas para clientes idosos que procuram os Caps-AD (NORONHA et al., 2019). O conhecimento sobre o uso de drogas por idosos e as questões que contribuem para o início tardio ou manutenção do vício na vida precisarão ser continuamente atualizados (KUERBIS et al., 2014). Outro agravante fator de confundimento, conforme apontam Fernandes et al. (2017), o número de pessoas nessa faixa etária que busca assistência de saúde em serviços especializados para o tratamento do uso de substâncias psicoativas tem sido disseminadamente muito baixo.

Existem algumas características importantes que precisam ser mantidas em mente ao lidar com o uso de drogas por pessoas idosas. Como um idoso tem maior probabilidade de sofrer de situações estressantes relacionadas à idade, é mais provável que ele use drogas para lidar com esses problemas (DEL'OLMO; CERVI, 2017). Amarante (2007) afirma que os problemas de uso de drogas são frequentemente confundidos com os sintomas de depressão, demência ou outros transtornos associados ao envelhecimento. Com efeito, a legitimidade das pesquisas sobre consumo de drogas tem, reiteradamente, sofrido críticas que, comumente, não se fundamentam, porquanto o objetivo de qualquer pesquisa epidemiológica é alcançar a realidade sobre um dado fenômeno. Quando se trata de consumo de substâncias psicoativas, o receio em declarar um comportamento que é revestido de preconceitos, certamente, resulta um universo subestimado, que não pode ser confundido com a esfera de alcance da pesquisa (FERRAZ, 2005).

A prevenção voltada para o uso abusivo e/ou dependência de álcool e outras drogas pode ser definida como um processo de planejamento, implantação e implementação de múltiplas estratégias voltadas para a redução dos fatores de risco específicos e fortalecimento dos fatores de proteção (HIRDES et al., 2015). Implica necessariamente a inserção comunitária das práticas propostas, com a colaboração de todos os segmentos sociais disponíveis. A prevenção teria como objetivo impedir o uso de substâncias psicoativas pela primeira vez, impedir uma escalada do uso e minimizar as consequências de tal uso (BODSTEIN; LIMA; BARROS, 2014). As estratégias de prevenção devem contemplar a utilização combinada dos seguintes elementos: fornecimento de informações sobre os danos do álcool e outras drogas, alternativas para lazer e atividades livres de drogas; devem também facilitar a identificação de problemas pessoais e o acesso ao suporte para tais problemas. Devem buscar principalmente o fortalecimento de vínculos afetivos, o estreitamento de laços sociais e a melhora da autoestima das pessoas.

Entre o aumento da prevalência do uso de drogas ilícitas entre idosos e os desafios associados à identificação e tratamento dos problemas de uso, fica evidente que o sistema de saúde como um todo não está bem preparado para as mudanças que são esperadas nos próximos anos, como reflexo da COVID-19 (SALCEDOBARRIENTOS, Dora Mariela; SILVA PAIVA, Maria Vitória; SILVA, 2020). Devem ser feitos esforços para desenvolver instrumentos eficazes de triagem do uso e para melhorar o conhecimento dos profissionais de saúde e o tratamento do uso e abuso de drogas lícitas/ilícitas entre os idosos.

\section{CONSIDERAÇÕES FINAIS}

O perfil etário dos pacientes atendidos nos Caps$\mathrm{AD}$ onde o estudo foi realizado, a variação de idade foi de 60 a 76 anos. Observou-se, também, um maior percentual de representação masculina, sem companheiro(a), com ensino fundamental incompleto e desempregados.

A uso de drogas lícitas e ilícitas entre idosos ainda é um assunto subestimado e desconhecido pela sociedade e, por grande parte dos profissionais de saúde, em decorrência de todos os rótulos associados à terceira idade. Os impactos a saúde física e mental causados pelo uso de drogas são severos em idosos, mesmo em quantidades mínimas, sendo o álcool a droga mais utilizada seguida do tabaco, crack, maconha, êxtase, cocaína, anfetamina, benzodiazepínicos e demais drogas. Esse cenário robustece a necessidade de resgatar a autonomia desses pacientes, permitindo sua reinserção na comunidade, através do trabalho com valor social, da busca dos direitos de cidadania a partir de uma assistência especializada, individualizada e que promova melhoria da qualidade de vida dessa população e de seus familiares.

Por fim, os transtornos relacionados a esse uso entre idosos ainda é pouco abordado e conhecido, no entanto, com tendência de um crescimento substancial nos próximos anos com a pandemia de COVID-19; que causa o uso de drogas lícitas e/ou ilícitas de forma 
crescente e de maneira silenciosa, e irá aumentar paralelamente ao crescimento dessa população, sendo, por conseguinte, já é considerado um grave problema de saúde pública.

\section{Agradecimentos}

A Fundação de Amparo à Pesquisa do Estado do Rio de Janeiro - FAPERJ e ao Conselho Nacional de Desenvolvimento Científico e Tecnológico - CNPq.

\section{REFERÊNCIAS}

ALMEIDA, José Miguel Caldas. Política de saúde mental no Brasil: o que está em jogo nas mudanças em curso. Cadernos de Saúde Pública, v. 35, n. 11, e00129519, 2019. https://doi.org/10.1590/0102311X00129519

AMARANTE, Paulo. Saúde mental e atenção psicossocial. Rio de Janeiro: Fiocruz, 2007.

BAPTISTA, Makilim Nunes; CAMPOS, Dinael Corrêa. Metodologias de Pesquisa em Ciências: análises quantitativa e qualitativa. - 2. ed. - Rio de Janeiro: LTC, 2016.

BEYNON, Caryl M., McVEIGH, Jim; ROE, Brenda. Problematic drug use, ageing and older people: trends in the age of drug users in northwest England. Ageing and Society, v. 27, n. 6, p. 799-810, 2007. https://doi.org/10.1017/S0144686X07006411

BODSTEIN, Airton; LIMA, Valéria Vanda Azevedo; BARROS, Angela Maria Abreu. A vulnerabilidade do idoso em situações de desastres: necessidade de uma política de resiliência eficaz. Ambiente \& Sociedade, v.17, n.2, p.157-174, 2014. https://doi.org/10.1590/S1414-753X2014000200011

BRASIL. Lei $\mathrm{n}^{\circ} 10.216$, de 6 de abril de 2001. Dispõe sobre a proteção e os direitos das pessoas portadoras de transtornos mentais e redireciona o modelo assistencial em saúde mental. Diário Oficial da União, 2001. Disponível em: https://hpm.org.br/wpcontent/uploads/2014/09/lei-no-10.216-de-6-de-abril-de2001.pdf

BRASIL. Ministério da Saúde. Conselho Nacional de Saúde. Resolução No 466, de 12 de dezembro de 2012. Disponível

em: https://www.iesb.br/Cms_Data/Contents/Portal/Media/ar quivos/466.pdf

CASTRO-COSTA, Érico; FERRI, Cleusa Pinheiro;
LIMA-COSTA, Maria Fernanda Furtado; ZALESKI, Marcos; PINSKY, Ilana et al. Alcohol consumption in late-life - The first Brazilian National Alcohol Survey (BNAS). Addictive Behaviors, v. 33, n. 12, p. 1598-1601, 2008. https://doi.org/10.1016/j.addbeh.2008.07.004.

CRUZ, Vânia Dias; SANTOS, Silvana Sidney Costa; GAUTÉRIO-ABREU, Daiane Porto; SILVA, Bárbara Tarouco; ILHA, Silomar. Consumo de drogas entre pessoas idosas e a redução de danos: reflexão a partir da complexidade. Escola Anna Nery, v. 20, n. 3, e20160076, 2016. https://doi.org/10.5935/14148145.20160076

DEL'OLMO, Florisbal de Souza; CERVI, Taciana Marconatto Damo. Sofrimento mental e dignidade da pessoa humana: os desafios da reforma psiquiátrica no Brasil. Sequência, n. 77, p. 197-220, 2017. https://doi.org/10.5007/2177-7055.2017v38n77p197

DESTRO, José Stéfano Faia. Dependência de substâncias psicoativas entre idosos: um desafio para a saúde pública. Regrad, v. 11, n. 1, p. 1-15, 2018. Disponível em: https://revista.univem.edu.br/REGRAD/article/view/259 2

FÁVERO, Luiz Paulo Lopes; BELFIORE, Patrícia Prado; SILVA, Fabiana Lopes; CHAN, Betty Lilian. Análise de dados: modelagem multivariada para tomada de decisões. Rio de Janeiro: Elsevier, 2009.

FEITOSA, Ana Nery de Castro; FERREIRA, Leandro de Andrade; OLIVEIRA, Adriano Monteiro; FEITOSA, José Kleidir de Castro. O uso de substâncias psicoativas em idosos. Revista Multidisciplinar e de Psicologia, v.10, n. 30, p. 245-251, 2016. Disponível em: https://idonline.emnuvens.com.br/id/article/view/473/583

FERNANDES, Sara Silva; MARCOS, Cristiane Barros; KASZUBOWSKI, Erikson; GOULART, Leonardo Salomão. Evasão do tratamento da dependência de 
drogas: prevalência e fatores associados identificados a partir de um trabalho de Busca Ativa. Cadernos Saúde Coletiva, v. 25, n. 2, p. 131-137, 2017. https://doi.org/10.1590/1414-462X201700020268

FERRAZ, Sabrina Mendes. Estudo da prevalência de uso de substâncias psicoativas por enfermeiros. Dissertação de Mestrado, Escola de Enfermagem da Universidade Federal de Minas Gerais. Belo Horizonte, 2010.

FERREIRA, Aline Cristina Zerwes; BORBA, Letícia de Oliveira; CAPISTRANO, Fernanda Carolina; CZARNOBAY, Juliana; MAFTUM, Mariluci Alves. Fatores que interferem na adesão ao tratamento de dependência química: percepção de profissionais de saúde. Revista Mineira de Enfermagem, v 19, n. 2, p. 150156, 2015. Disponível em: https://pesquisa.bvsalud.org/portal/resource/pt/lil-768602

GIL, Antônio Carlos. Como elaborar projetos de pesquisa. 4. ed. São Paulo: Atlas, 2008.

GONÇALVES, Teresa Sequeira; NUNES, Marilene Rivany. Perfil dos usuários do Centro de Atenção Psicossocial Álcool e Drogas. Perquirere, v.11, n.2, p. $169-178,2014$.

HIRDES, Alice; MARCON, Grasiela; BRANCHI, Rafael Nicola; VIVIAN, Aline Groff. Prevenção ao uso de álcool e outras drogas e tratamento na Atenção Primária à Saúde em um município do Sul do Brasil. Aletheia, v. 46, p. 74-89, 2015. https://doi.org/10.29327/226091

KUERBIS, Alexis; SACCO, Paul; BLAZER, Dan G.; MOORE, Alison A. Substance abuse among older adults. Clinics in Geriatric Medicine, v. 30, n. 3, p. 629654, 2014. https://doi.org/10.1016/j.cger.2014.04.008

MIRANDA, Gabriella Morais Duarte; MENDES, Antônio da Cruz Gouveia; SILVA, Ana Lucia Andrade. O envelhecimento populacional brasileiro: desafios $\mathrm{e}$ consequências sociais atuais e futuras. Revista Brasileira de Geriatria e Gerontologia, v. 19, n. 3, p. 507$519,2016.2 \quad$ https://doi.org/10.1590/180998232016019.150140

MOORE, David. A Estatística Básica e sua Prática. Rio de Janeiro: LTC, 2000.
NORONHA, Beatriz Prado; NASCIMENTO-SOUZA, Mary Anne; LIMA-COSTA, Maria Fernanda; PEIXOTO, Sérgio Viana. Padrões de consumo de álcool e fatores associados entre idosos brasileiros: Pesquisa Nacional de Saúde (2013). Ciênc. saúde coletiva, v. 24, n. 11, p. 4171-4180, 2019. https://doi.org/10.1590/1413812320182411.32652017

PELISOLI, Cátula da Luz; MOREIRA, Ângela Kunzler. Avaliação de um centro de atenção psicossocial por meio do perfil de seus usuários. Mental, v.5, n.8, p. 61$75,2007$.

PILLON, Sandra Cristina; CARDOSO, Lucilene; PEREIRA, Gisela Amorim Marques; MELLO, Emmanuel. Perfil dos idosos atendidos em um centro de atenção psicossocial: álcool e outras drogas. Escola Anna Nery, v. 14, n. 4, p. 742-748, 2010. https://doi.org/10.1590/S1414-81452010000400013

ROE, Brenda; BEYNON, Caryl; PICKERING, Lucy, DUFFY, Paul. Experiences of drug use and ageing: health, quality of life, relationship and service implications. Journal of Advanced Nursing, v. 66, n. 9, p. 1968-1979, 2010. https://doi.org/10.1111/j.13652648.2010.05378.x.

SALCEDO-BARRIENTOS, Dora Mariela; SILVA PAIVA, Maria Vitória; SILVA, André Luiz Pereira. Terapia Comunitária Integrativa para idosos em plataforma virtual durante a pandemia associada a COVID-19. Temas em Educação e Saúde, v. 16, n. esp. 1, p. 360-375, 2020. https://doi.org/10.26673/tes.v16iesp.1.14317

SANTANA, Ramaile Tomé; MIRALLES, Nathali Carmel Weiler; ALVES, Jéssica Freitas, SANTOS, Vanessa Ávila; VINHOLES, Ubirajara et al. Perfil dos usuários de CAPS-AD III. Brazilian Journal of health Review, v. 3 , n. $1, \quad$ p.1343-1357, 2020. https://doi.org/10.34119/bjhrv3n1-103

SANTOS, Deivisson Vianna Dantas; FEDERHEN, Chayanne; SILVA, Thiago Augusto; SANTOS, Isabella Renata;

LEVINO, Caroline de Azevedo et al. A gestão autônoma da medicação em Centros de Atenção Psicossocial de Curitiba (PR). Saúde em Debate, v. 44, n. 3, p. 170-183, 2020. https://doi.org/10.1590/0103-11042020E315 
SILVA, Jerto Cardoso; HERZOG, Lísia Mânica. Psicofármacos e psicoterapia com idosos. Psicologia \& Sociedade, v. 27, n. 2, p. 438-448, 2015. https://doi.org/10.1590/1807-03102015v27n2p438

TREVISAN, Erika Renata; CASTRO, Sybelle de Souza. Centros de Atenção Psicossocial - álcool e drogas: perfil dos usuários. Saúde em debate, v.43, n.121, p.450463, 2019. https://doi.org/10.1590/0103-1104201912113

WANG, Yuan-Pang; ANDRADE, Laura Helena. Epidemiology of alcohol and drug use in the elderly. Current opinion in psychiatry, v. 26 , n. 4 , p. 343-348,
2013. https://doi.org/10.1097/YCO.0b013e328360eafd

WORLD HEALTH ORGANIZATION (WHO). Global strategy to reduce the harmful use of alcohol. Geneva: WHO; 2013.

ZHANG, Ruiyuan, SHEN, Luqi; MILES, Toni; SHEN, Ye; CORDERO, Jose et al. Association of low to moderate alcohol drinking with cognitive functions from middle to older age among US adults. JAMA, v. 3, n. $6, \quad$ e207922, 2020. https://doi.org/10.1001/jamanetworkopen.2020.7922 